\title{
Experimental Confirmation of Radiative Corrections to Bremsstrahlung and Pair Production at High Energies
}

\author{
H. D. Schulz AND G. Lutz \\ Deutsches Elektronen-Synchrotron, Hamburg, Germany
}

(Received 10 July 1967)

\begin{abstract}
Radiative corrections to bremsstrahlung and pair-production cross sections have been detected experimentally by measuring the thin-target bremsstrahlung spectrum of 5-GeV electrons with a pair spectrometer. Deviations of up to $5 \%$ from lowest-order calculations have been observed and found to agree with predictions for radiative corrections given by Mork and Olsen.
\end{abstract}

\section{INTRODUCTION}

$\mathrm{R}^{\mathrm{s}}$ ECENTLY Mork and Olsen ${ }^{1}$ calculated the radiative corrections to bremsstrahlung and pairproduction cross sections, integrated over all angles at high energies. These calculations offer the possibility of comparing theoretical predictions of higher-order effects with experimental results. For this purpose we calculated radiative corrections using the method of MO for an experiment in which the bremsstrahlung spectrum produced by $5-\mathrm{GeV}$ electrons in a thin target was measured with a pair spectrometer. In this case the counting rate at fixed photon energy will be proportional to the product of the bremsstrahlung and pair-production cross sections, and the sum of higherorder corrections to these two cross sections should show up as the difference between measured points and lowest-order calculations.

\section{THEORY}

MO start out with the radiative corrections calculated by Brown and Feynman ${ }^{2}$ for the Compton effect; these are transferred into corrections to the bremsstrahlung cross section via the Weizsäcker-Williams method. The contribution by the vacuum polarization graph has no counterpart in the Compton process; it is calculated directly for bremsstrahlung and is shown to be negligible compared to the contribution from the other higher-order graphs.

The results are given in the form

$$
\begin{aligned}
d \sigma^{B} & =d \sigma_{0}{ }^{B}\left(1+\delta^{B}\right), \\
\delta^{B} & =F_{1}(x)+F_{2}(x) \ln \left(\Delta k / E_{0}\right), \quad x=k / E_{0},
\end{aligned}
$$

where $d \sigma_{0}{ }^{B}$ is the lowest-order bremsstrahlung cross section and $d \sigma^{B}$ the bremsstrahlung cross section including radiative corrections, $E_{0}$ the primary energy of the electrons, and $k$ the energy of the emitted photon. $\Delta k$ is the maximum energy available for the emission of a second photon in the same bremsstrahlung process. $F_{1}$ and $F_{2}$ are given in Table I as functions of the relative photon energy $x$.

The validity of formula (1) is limited by the condition $\Delta k \ll E_{0}$. In our case the outgoing electron is not

\footnotetext{
${ }^{1}$ K. Mork and H. Olsen, Phys. Rev. 140, B1661 (1965); will be cited as $\mathrm{MO}$ in the following.

${ }^{2}$ L. M. Brown and R. P. Feynman, Phys. Rev. 85, 231 (1952).
}

detected, and $\Delta k$ is given by $\Delta k=E_{0}-k$; thus the $\Delta k$ will assume values which are comparable to $E_{0}$. Therefore we applied the correct expression for the doublebremsstrahlung cross section for energies of the second photon greater than $0.02 E_{0}$ :

$$
\begin{aligned}
\delta^{B}=F_{1}(x)+ & F_{2}(x) \ln \left(\Delta k / E_{0}\right) \\
& +\int_{\Delta k}^{E_{0}-k} \frac{d \sigma_{D}{ }^{B}}{d k_{1}}\left(\frac{d \sigma_{0}{ }^{B}}{d k}\right)^{-1} d k_{1}, \quad \Delta k \ll E_{0} .
\end{aligned}
$$

$k_{1}$ is the energy of the additional photon, $d \sigma_{D}{ }^{B}$ is the cross section for double emission of bremsstrahlung given as a three-dimensional integral by MO. This integral was evaluated numerically and agrees for small $k_{1}$ with $d \sigma_{0}^{B}\left(F_{2} / k_{1}\right) d k_{1}$ as derived from (1). The resulting correction $\delta^{B}$ is plotted in Fig. 1.

The radiative correction for the pair-production cross section is obtained from that of the bremsstrahlung process by applying the substitution rule

$$
E_{0} \rightarrow-E^{+}, \quad k \rightarrow-k, \quad d k / k \rightarrow\left(E^{+}\right)^{2} d E^{+} / k^{3} .
$$

The correction for pair production then reads

$$
\begin{aligned}
d \sigma^{P} & =d \sigma_{0}{ }^{P}\left(1+\delta^{P}\right), \\
\delta^{P} & =G_{1}(\epsilon)+G_{2}(\epsilon) \ln (\Delta k / k), \quad \epsilon=E^{+} / k,
\end{aligned}
$$

subject to the condition $\Delta k \ll k$. Again $\Delta k$ is the maximum possible energy of an additional photon emitted in the pair-production process. $G_{1}$ and $G_{2}$ were tabulated by MO.

When the energy of the bremsstrahlung photons is determined with a pair spectrometer, pair-production processes involving the emission of an additional photon will contribute to the counting rate of pairs of energy below that of the original photon. The difference in

TABLE I. $F_{1}$ a and $F_{2}$ as functions of the relative photon energy $x=k / E_{0}$.

\begin{tabular}{ccccccccc}
\hline$x$ & 0.1 & 0.2 & 0.3 & 0.4 & 0.5 & 0.6 & 0.7 & 0.8 \\
\hline $100 F_{1}$ & 0.10 & 0.19 & 0.30 & 0.43 & 0.60 & 0.81 & 1.08 & 1.50 \\
$100 F_{2}$ & 0.02 & 0.05 & 0.10 & 0.16 & 0.25 & 0.35 & 0.49 & 0.70 \\
$x$ & 0.9 & 0.95 & 0.96 & 0.97 & 0.98 & 0.99 & 0.995 & \\
\hline $100 F_{1}$ & 2.30 & 3.25 & 3.60 & 4.07 & 4.79 & 6.18 & 7.78 & \\
$100 F_{2}$ & 1.04 & 1.37 & 1.48 & 1.62 & 1.81 & 2.14 & 2.46 &
\end{tabular}

${ }^{a}$ Our values of $F_{1}$ differ from those given in Ref. 1. We thank Professo Olsen and Dr. Mork for kindly rechecking them. 


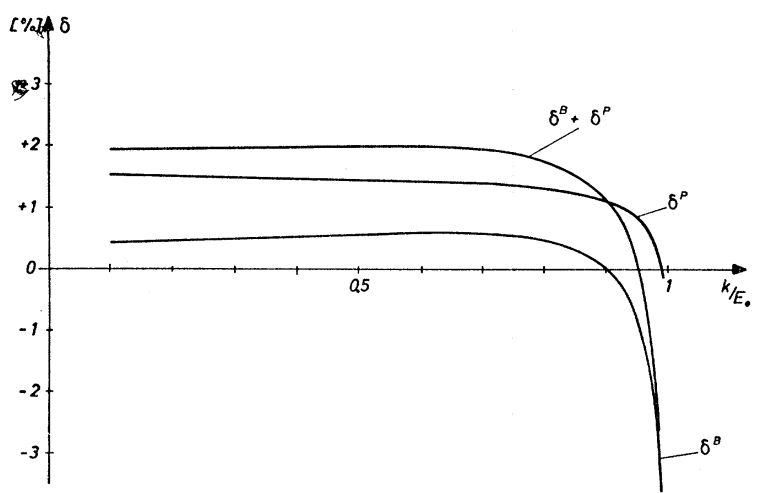

FIG. 1. Calculated effect of radiative corrections on the bremsstrahlung spectrum as measured with a pair spectrometer using symmetrical pairs only. $\delta^{B}$, contribution from the bremsstrahlung process; $\delta^{P}$, contribution from the pair-production process.

energy is equal to the energy of the additional photon that is not detected. Thus all photons having energies higher than the pair spectrometer setting can contribute to the counting rate at this setting via radiative pair production, and the influence of radiative corrections on pair production becomes dependent on the shape of the bremsstrahlung spectrum. One has to integrate over the probability of all such possible processes to obtain the correction for the counting rate at any point within the spectrum:

$$
\begin{aligned}
\delta^{P}=G_{1}+G_{2} \ln \frac{\Delta k}{k}+\int_{\Delta k}^{E_{0}-k} & \frac{d \sigma_{D}{ }^{P}}{d k_{1}}\left(\frac{d \sigma_{0}{ }^{P}}{d k}\right)^{-1} \\
& \times \frac{d n\left(k+k_{1}\right)}{d n(k)} d k_{1}, \quad \Delta k \ll k .
\end{aligned}
$$

$d \sigma_{D}{ }^{P}$ is the cross section for pair production with emission of an additional photon $k_{1}$ (see MO). This expression was evaluated in complete analogy to (2). The correction $\delta^{P}$ was applied to our experiment for $\epsilon=0.5$ and is plotted in Fig. 1.

The sum of $\delta^{B}$ and $\delta^{P}$ (Fig. 1) is the expected deviation of experimental points from lowest-order calculations, if the bremsstrahlung spectrum is measured with a pair spectrometer using symmetrical pairs only.

Deviations of up to $3 \%$ are predicted, but none had been observed so far. ${ }^{3}$ We have therefore checked the predicted higher-order effects by measuring a bremsstrahlung spectrum with maximum energy at $5.15 \mathrm{GeV}$ by means of a pair spectrometer.

Several requirements must be fulfilled for the observed deviations from lowest-order cross sections to be identifiable as radiative-correction effects:

(1) It must be shown that the neglect of radiative corrections is actually the dominating error in the "lowest-order" cross sections used here for comparison.

${ }^{3}$ For an experiment on radiative corrections to the positronelectron scattering cross section see A. Browman, B. Grossetete, and D. Yount, Phys. Rev. 151, 1094 (1966).
(2) No uncontrolled sources of experimental deviations are allowed that might mask the effect we are looking for.

The lowest-order cross sections used here for comparison with experimental data are the Bethe-Heitler formula with Coulomb correction ${ }^{4}$ for processes in the field of atomic nuclei, and the formula of Wheeler and Lamb $^{5}$ for processes in the field of atomic electrons. In both of these formulas screening is accounted for by using the Thomas-Fermi model of the atom. Apart from the neglect of radiative corrections, the uncertainties in these cross sections are due to the following approximations.

Static approximation and neglect of Compton graphs involve a relative error on the order of $(q / M c)^{2}$, where $q$ is the recoil momentum transferred to the target particle, and $M$ is its mass. ${ }^{6}$ Since more than $90 \%$ of the recoils in the cross sections integrated over the angles lie below $1 m c$ ( $m=$ electron mass), this error is negligible for processes in the field of the nucleus but not necessarily for processes on target electrons. Here additional exchange graphs have to be taken into account. According to Suh and Bethe, ${ }^{7}$ and Mork, ${ }^{8}$ however, the combined effect of the Compton and exchange graphs on the cross section for processes on target electrons is negligible at high energies; it contributes an error of less than $1 \%$ to the Wheeler-Lamb cross section in our case. Since this error enters into the total atomic cross section with a factor $1 / Z$ only, high- $Z$ material should be chosen for the targets so as to keep the total error negligible. The use of the Thomas-Fermi model introduces an error of less than $0.2 \%$ for $Z>20$. The errors due to the Coulomb correction, to the neglect of nuclear form factors, ${ }^{9}$ and to the neglect of nuclear magnetic moments are all below $0.1 \%$, and are thus negligible in this context.

Compared to the contribution of radiative corrections, these errors are indeed small at high energies for $Z>20$.

\section{EXPERIMENTAL ARRANGEMENT}

Deviations due to the experimental arrangement were kept at a negligible level by the following measures: For measuring pure thin-target spectra, a thin titanium foil of $80 \mu$ thickness ( $=2 \times 10^{-3}$ radiation lengths) was used as the bremsstrahlung target within the electron synchrotron. Multiple traversals through the target were cut down by thick secondary targets in suitable positions inside the synchrotron ring. A mean rate of

\footnotetext{
${ }^{4}$ Bremsstrahlung: H. Olsen, Phys. Rev. 99, 1335 (1955); pair production: H. Davies, H. A. Bethe, and L. C. Maximon, ibid. 93, 788 (1954).

${ }_{5}^{5}$ J. A. Wheeler and W. E. Lamb, Phys. Rev. 55, 860 (1939); 101, 1836 (1956).

${ }^{6}$ J. D. Bjorken, S. D. Drell, and S. C. Frautschi, Phys. Rev. 112, 1309 (1958).

${ }^{7} \mathrm{~K}$. S. Suh and H. A. Bethe, Phys. Rev. 115, 672 (1959).

8 K. J. Mork, Phys. Rev. 160, 1065 (1967).

${ }^{9}$ B. J. Malenka and H. P. Smith, Phys. Rev. 132, 863 (1963).
} 

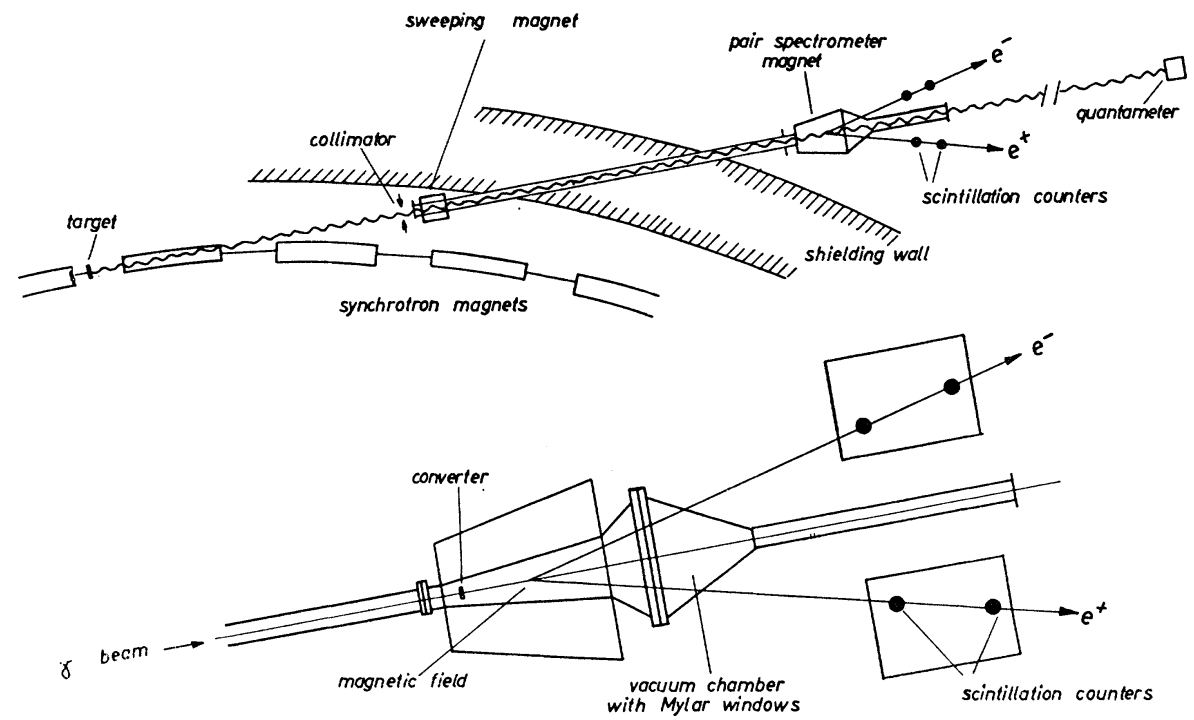

Fig. 2. Experimental setup.

only one traversal was derived by calculation from the electron current in the synchrotron, from the target thickness, and from the photon yield. The collimation angle was fixed at 5 times the elementary emission angle $m / E_{0}$ in order to ensure integration over the photon emission angles. For this configuration the effect of collimation on the photon spectrum was calculated to be very small and opposite to the effect expected from the radiative corrections. The primary divergence of electrons in the synchrotron helps in mixing emission angles and improving the integration over the angles.

Beam layout and pair spectrometer are shown in Fig. 2. A silver foil of $10 \mu$ thickness $\left(=1 \times 10^{-3}\right.$ r. 1.) served as the conversion target inside the pair-spectrometer magnet. Counter telescopes in fixed positions behind the magnet detected symmetrical pairs only. The design value of the momentum acceptance $\Delta p / p$ of the counter telescopes was chosen to be $2 \%$. The actual value of $\Delta p / p$ was determined with an estimated relative error of $\pm 2 \%$ from the geometrical width of the momentum defining scintillator and from particle trajectories, in the calculations of which the measured magnetic field distribution was taken into account.

The energy of the accepted pairs was varied by changing the current of the spectrometer magnet. For photon energies above $1.5 \mathrm{GeV}$ the energy resolution of the pair spectrometer is almost identical with the momentum acceptance of the counters, since at these energies the mean opening angle of pairs is small compared to the angular acceptance of the counters.

The detection efficiency of the pair spectrometer has been calculated to be better than $99.5 \%$ above $2.5-\mathrm{GeV}$ photon energy. In the calculation the following items were taken into account: photon beam size at the converter target, production angular distribution of pair electrons, scattering of electrons within the converter and telescope counters, and geometrical size of the counters.

A quantameter of the Wilson type served as a beam monitor; its calibration constant had been determined by comparison with a Faraday cup in the external electron beam of DESY. ${ }^{10}$ The error in the quantameter constant is estimated at $1 \%$; the error in the momentum acceptance of the counter telescopes, the second power of which enters into the counting rate, is about $2 \%$; uncertainties in weight and homogeneity of the converter target, maximum photon energy, and detection efficiency at energies above $2.5 \mathrm{GeV}$ contribute an error of less than $1 \%$. This implies that any absolute determination of pair-production cross sections with this arrangement cannot have an error smaller than $3 \%$, which is not sufficient for determining higher-order effects by absolute measurement; but the effect of radiative corrections will also change the apparent form of the bremsstrahlung spectrum, and relative measurements can be made with a much higher precision. This is especially true in the high-energy region above 2.5 $\mathrm{GeV}$, where no energy-dependent corrections must be applied. Then the error is limited only by the stability of the apparatus $(0.5 \%)$, and by the statistics of counting. Thus an error of approximately $0.7 \%$ is attainable, which is sufficient to detect radiative-correction effects. We are then, to within $3 \%$, free to choose a common normalization factor for all the measured points in order to compare them with the theoretical spectrum in the lowest order.

\section{RESULTS}

Figure 3 shows the results of a measurement of the bremsstrahlung spectrum for $5.15-\mathrm{GeV}$ electron energy in comparison with the theoretical spectrum, using

${ }^{10} \mathrm{~A}$. Ladage and H. Pingel, DESY-Bericht Report No. $65 / 12,1965$ (unpublished). 


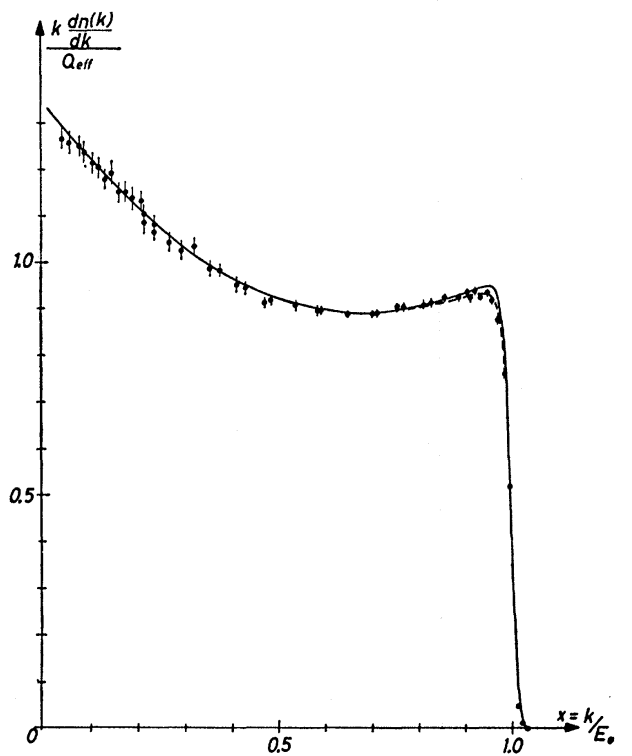

FIG. 3. Bremsstrahlung spectrum at $5.15-\mathrm{GeV}$ electron energy. Solid curve, theoretical spectrum without radiative corections; dashed curve, theoretical spectrum with radiative corrections. Theoretical spectra folded with the experimental resolution and normalized to the same value at $x=0.8$.

lowest-order cross sections for bremsstrahlung and pair production. The theoretical curve takes into account the energy spread of primary electrons in the synchrotron during spillout, as well as the finite energy resolution of the pair spectrometer. The experimental data have been corrected for background $(<1 \%)$ and accidentals (about 10\%); the latter were measured simultaneously. Experimental points below $x=0.4$ have been further corrected for the calculated detection efficiency of the pair spectrometer. The estimated error of this procedure is included in the error bars shown at each point. Experimental points were normalized to fit the theoretical curve between $x=0.5$ and 0.8 , since in this region the radiative corrections are almost independent of photon energy (see Fig. 1). The normali-

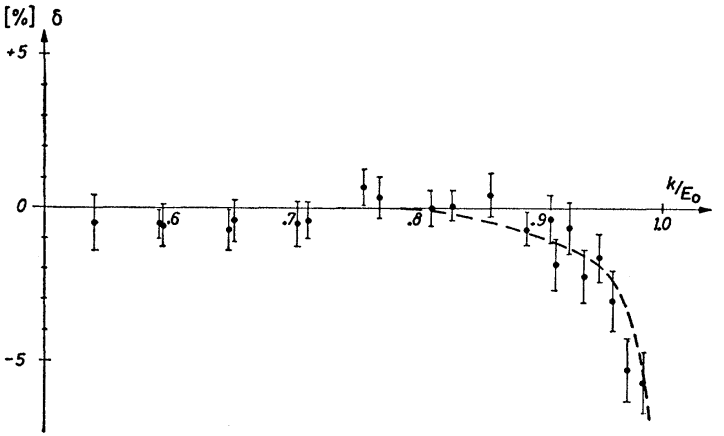

FIG. 4. Relative difference between experimental points and the theoretical bremsstrahlung spectrum (Refs. 4 and 5 ) folded with the experimental resolution. Dashed curve, deviations expected from radiative corrections.

zation required the application of a factor of 0.97 to all experimental points.

Except for the region $0.8<x<1.0$ there is good agreement between the experimental points and the theoretical curve. Deviations above $x=0.8$ are plotted in Fig. 4 and compared with the deviations predicted by radiative-correction effects. Within the experimental error there is satisfactory agreement between the predicted and measured deviations from lowest-order calculations.

Two conclusions can be drawn from this result: (1) The predictions by Mork and Olsen ${ }^{1}$ on radiative corrections to bremsstrahlung and pair production are experimentally confirmed. (2) Within an experimental error of $1 \%$, no deviations of the shape of the bremsstrahlung spectrum from the predictions of quantum electrodynamics including higher-order effects have been observed.

\section{ACKNOWLEDGMENTS}

We thank Professor Jentschke for initiating this work and Professor Olsen and Dr. Mork for communicating their results prior to publication. The unfailing cooperation of the synchrotron staff and the computer center is gratefully acknowledged. 